\title{
The use of avatars in digital role-playing games (RPGs) in computer-assisted language learning (CALL)
}

Charly Harbord Euan Dempster

Darshana Jayemanne

Harbord, C., Dempster, E. \& Jayemanne, D. (2021) 'The use of avatars in digital role-playing games (RPGs) in computer-assisted language learning (CALL)'. In M. Peterson, K. Yamazaki \& M. Thomas (eds.) Digital games and language learning : theory, development and implementation. Bloomsbury Academic, London, pp. 137-162.

DOI: https://doi.org/10.5040/9781350133037.ch-007 


\title{
Chapter 7
}

\section{The use of Avatars in Digital Role-Playing Games (RPGs) in Computer-Assisted Language Learning (CALL)}

\author{
Charly Harbord and Euan Dempster
}

\section{Introduction}

This chapter provides an overview of the relationship between digital games and second language acquisition (SLA). The discussion examines the major game types utilized in computer-assisted language learning (CALL) and then focuses on providing a theoreticallyinformed rationale for the use of digital role-playing games (RPGs) to enhance second language learning in conjunction with the material used within the classroom. The chapter reports on an ongoing learner-based project in which a purpose-built RPG has been created to investigate the role of this type of digital game in facilitating second language acquisition. This chapter further reports on a small-scale pilot project that forms part of the above research. This investigates the potential effects of avatar choice on perceptions of learning in a group of Chinese EFL learners. The findings on learner avatar preferences are examined, demonstrating the relevance of 'the thin-slice effect' (Shin et al. 2019) in learner's avatar choice. With this bias, players judge other avatars based on how honestly they have represented themselves as an avatar. This would seem to imply that if the player has chosen an avatar that is more culturally representative of the target language (TL), they would assume the same of other players' avatars. The chapter closes with a discussion of the influence of the findings on the wider research into the use of RPGs in CALL and identifies areas of likely interest for future research. 


\section{Background}

The ideas behind this chapter originally came from the literature on the use of digital games in CALL (Peterson 2013; Reinhardt 2019) and from the personal experiences of one of the authors who observed her son learning Japanese through playing games on a regionally locked Nintendo 3DS. As a language teacher and gamer, this author was more than aware of the difference between the motivation to do homework and the motivation to play. Additionally, whilst working as an English foreign language teacher in China the above author noted how the level of English produced in the classroom compared to the astonishingly fluent language produced during the breaks from the students who went to play games online. It appeared that the motivation derived from gaming had aided the students in learning English without them even realising it (implicit learning).

The notion that implicit learning was taking place was further evidenced when teaching on a masters of professional practice orientation programme (MPPOP) in Beijing. This afforded the creation of a close working relationship and knowledge exchange with the School of Design and Informatics from Abertay University who supplied lecturing staff. It is a programme where games design, professional behaviours and English for Specific Academic Purposes (ESP) were combined in order to create the complete Scottish university preparatory experience. Thereby creating a unique opportunity for research into SLA and games, especially as the students involved were both English as second language learners and designers and players familiar with RPGs and MMORPGs. Again, the disparity between the English language produced within the classroom activities and the language produced in games design discussions and gameplay appeared highly significant. The following discussion provides an overview of the relationship between gaming and second language acquisition (SLA). 


\section{Digital Games and SLA}

There is a distinct difference between 'second language acquisition'; characterised by the implicit gaining of a second language knowledge through gradual exposure and 'second language learning', characterised by the conscious learning of a second language through explicit teaching (Krashen 1989). The Confucian style of language education implemented in China prescribes rote repetition and memorisation of grammatical functions, creating a disconnect to second language acquisition (Suzuki et al. 2019). This produces a mechanical understanding of the TL but not necessarily the ability to use it in a confident and improvised manner; for example, in real-life conversation (Mokhatar et al. 2017). Second language acquisition may be enhanced by active participation in activities with structured goals in the TL. Activities such as group quests, team battles and in game interactions, including those with non-player characters (NPCs), foster the immersion and interactivity of the game. These form the basic aspects of game mechanics, yet are so hard to replicate in a convincing and genuine manner within an educational setting (Cheng et al. 2017). Peer cooperation during RPG gameplay between native and second language learners has the ability to inform the collaborative scaffold around the language learning experience, with the TL and the game itself acting as a mediator. As result of paradigms shifts in modern education, L2 learners are now viewed as social beings as opposed to individual learners (Lee and Pass 2014). By working together with a native speaker to achieve a goal, the learner of the TL will progress further than they would have been able to do working by themselves (Zhang 2009).

According to Hadfield (1984) language games can be compartmentalised into two fields: linguistic and communicative. Games with a linguistic focus concentrate more on fluency and accurate grammar, whereas communicative games are aimed at building rapport and transference of information. As will be noted at a later stage of this discussion, the aim of this current research places the focus firmly on 'language for use' and the learner's ability to 
implement it into new contexts and situations (Ellis 2019; Suzuki et al. 2019). Motivation is intrinsic with regards to learning, but is an intangible concept which is challenging to quantify. Topirceanu (2017) created a motivational spectrum with a sliding scale of motivation. At one end of the spectrum there is less self-motivation and externally regulated motivational factors form the basis of decision-making and learning; for example, rote learning grammar for a test (a form of dependent learning). The opposite end of the sliding scale represents intrinsic motivation (autonomous learning), where a person carries out an action or learns something purely for the personal gain and enjoyment not due to outside pressure (Felicia 2011). This is the goal for educational games, where a student actively chooses to play the game and thereby learn outside the classroom context (Blumberg and Altschuler 2011). Games such as Minecraft and The SIMs may be seen to have crossed the education/fun divide. Both games have been used for educational and specifically second language research (Ames and Burrell 2017) and have proved valuable tools for such projects. However, it should be noted that whilst both of these games (and others like them) may be being used outside the research/educational scenario, it is challenging to prove if they are still being played within the educational context of the classroom/research project. In the following section the discussion provides an overview of the major game types utilized in CALL.

\section{Major Game Types Utilized in CALL}

Research on CALL relating to gaming can be observed to focus on two main types of game: commercial off-the-shelf games (COTS) and serious games or games designed specifically for learning rather than for entertainment (Connolly et al. 2012). Both types of game have the potential to support effective language learning by increasing motivation, offering real time feedback and increasing exposure to the TL. The serious games genre focuses on an educational agenda first and entertainment second (Sorensen and Meyer 2007). One aspect 
that requires consideration when designing such games is the balance between formal and informal learning. Formal learning, such as that carried out within a school setting, primarily focuses on a quantifiable educational goal. Whereas informal learning, which often takes place outside of the classroom setting, focuses on entertainment first and gaining knowledge and/or abilities second.

There has been a growth in serious games development specifically designed to be educational first and entertaining second. Whilst these games have been successful within the classroom, they are seldom played in other contexts. A possible reason for this phenomenon may be due to an imbalance between language input and gameplay (Chen and Yang 2013; de Haan 2005). COTS are primarily designed for fun but may present learning opportunities almost by proxy. There is both anecdotal and research evidence that role-playing games (RPGs) and other socially interactive games have clearly demonstrated improvements in TL production and intercultural communication (Romero et al. 2015). Game-based learning follows constructivist principles in that the kinaesthetic methods of "learning by doing" or "active learning" often form the basis of gameplay (Yang 2012). Whilst it could be stated that the linguistic content and cultural knowledge offered by COTS games have the potential to be the equal of second language learning textbooks and curriculum, it should be noted that the primary function of CALL is to enhance and complement traditional language learning (Alyaz et al. 2017). The research of Sung et al. (2015) demonstrated that by combining digital and traditional language learning methods, player flow is increased leading to improved motivation, which in turn enhances the ability to problem solve and increases learning potential. Flow is the careful balance of task difficulty and player ability which gives enough challenge to make the game enticing but not so much that the player becomes frustrated and quits (Nylund and Landfors 2015). One genre of game that has shown benefits for education within the realm of CALL are RPGS. The discussion in the next section 
examines research findings that highlight several of the potential benefits of engaging learners in RPG-based gameplay.

\section{Utilizing RPGs to Facilitate Second Language Learning: Hypothesized Benefits}

The causal relationship between RPGs and language acquisition is one that is of great interest to researchers and educators (Peterson 2013). As Table 7. 1 shows, a number of features of RPGs are hypothesized as facilitating language learning. RPG gameplay often focuses on making meaning from riddles and quests as well as remembering information for use later, creating the experience of not only increasing vocabulary but also understanding the context of the language used. Previously, in-game communication was limited to text chat. However, now players are able to interact verbally with players from all around the world. This is coupled with real-time exchanges where the player must respond in a timely manner and has the ability to replay situations where the outcome was not satisfactory (Cornillie et al. 2012). Being able to self-study at one's own pace and the ability to replay any situation with the option to use subtitles mean that RPGs appear well suited to language learning (de Haan et al. 2010). Subtitles serve to consolidate and confirm information heard, enhancing the comprehension of the language via multimodal input (Bird and Williams 2002). Furthermore, with in-game text second language learners can see their speech pattern which in itself can help identify errors and allows focus on speech (Ellis 2019; Peterson 2010). The discussion in the remainder of this section provides an overview of key findings reported in the literature.

Table 7.1. Hypothesized Advantages of Uses RPGs in Language Education

\begin{tabular}{|l|l|l|}
\hline Features of RPGs & Hypothesized Advantages & Author(s) \\
\hline
\end{tabular}




\begin{tabular}{|c|c|c|}
\hline Individual game play & Learner centred interaction & de Haan et al. 2010 \\
\hline The use of avatars & $\begin{array}{l}\text { Anonymity, reduction of social } \\
\text { cues, reduced perceived threats to } \\
\text { face and anxiety. } \\
\text { Opportunities for cross cultural } \\
\text { interaction }\end{array}$ & Rankin et al. 2008 \\
\hline Immersive gameplay & Reduction of inhibitions & $\begin{array}{l}\text { McCreery et al. } \\
2012\end{array}$ \\
\hline Problem solving & $\begin{array}{l}\text { Provides deeper learning, } \\
\text { inductive reasoning }\end{array}$ & Felicia 2011 \\
\hline In game tutorials & $\begin{array}{l}\text { Provide a scaffolding system with } \\
\text { which the player can learn the } \\
\text { mechanics/language needed at } \\
\text { their own pace, repetition helps } \\
\text { the player to become familiar with } \\
\text { the TL. }\end{array}$ & de Haan 2005 \\
\hline Social aspects & $\begin{array}{l}\text { Team quests and guilds require } \\
\text { need for interaction, provide } \\
\text { community bases and social } \\
\text { bonds. }\end{array}$ & $\begin{array}{l}\text { Alyaz et al. 2017; } \\
\text { Peterson 2011; } \\
\text { Peterson } 2006\end{array}$ \\
\hline Game and peer feedback & $\begin{array}{l}\text { Real-time modification of TL } \\
\text { utterances }\end{array}$ & de Haan 2005 \\
\hline
\end{tabular}




\begin{tabular}{|l|l|l|}
\hline Motivation & Learning efficacy autonomy & Warshauer 2005 \\
& increased by gameplay and social & \\
interaction & Authentic experiences to practice & Gee 2005 \\
\hline Realistic situations/tasks & TL & Zhang et al. 2017 \\
\hline Interaction with native & Scaffolded language forum & Sylven and \\
\hline speakers & & Sundqvist 2012 \\
\hline
\end{tabular}

\section{Research Findings: Interactional Context}

Researchers who have investigated the use of RPGs in CALL argue that a major advantage of RPGs is the interactional context provided (Jabbari and Eslami 2018; Peterson 2011; 2012). As most RPGs are designed with a focus on the individual player, they provide opportunities for individualized immersion in a context that is feedback rich, low stress and that compels TL use (Kim 2018). Moreover, the online nature of these games provides opportunities for authentic collaborative interaction involving problem-solving in the TL with a wider range of interlocutors including native speakers, than may be found in many conventional language classrooms (Gee 2005). Moreover, game support features such as tutorials provide scaffolding and opportunities for L2 vocabulary learning (de Haan 2005). For the purposes of this research the lens has been narrowed to focus on text-based RPGs, and textual language learning. 
It is noted in the literature that whilst a relatively new area of research, there is strong evidence to support the value of implementing socially interactive games for language learning. RPGs provide an arena for deep learning through activities such as problem solving and inductive reasoning (Felicia 2011). The social interaction aspect provides exposure to the TL culture enabling these games to benefit language learners (Alyaz et al. 2017). Findings from research by Sylven and Sundqvist (2012) demonstrated that when compared to a nongaming control group, the 11 to 12-year-old participants who played more than five hours of MMORPG games have significantly larger L2 vocabularies. RPG's stimulate the need for real-time interactions without the real-life consequences that may result from making mistakes. A fundamental feature of real-time game-based interactions is immediate feedback. By finding out instantly when a mistake has been made, a learner is able to amend their response as if in a real-time interaction. This increases the suspension of belief and immersion within the game world. In-game tutorials provide an inbuilt scaffolding system. When a player first starts a game, they must progress through a series of basic levels in order to understand the specific controls and mechanics of the game. It is this repetition that helps the TL user become more familiar with the language through exposure and thereby facilitating gradual acquisition (de Haan 2005). Additionally, players form in game communities which create social bonds and which may lead to support of language learning within the group (Peterson 2006). Peer related teamwork and feedback can provide a positive atmosphere for language learning. These factors aid the development of communicative competence by providing access to a practice arena that may enhance learning.

\section{Research Findings: Affective Factors, Motivation}

Within many classroom settings, language learning can be seen to be teacher-centric, where one teacher is in control of multiple students' learning progression (Topirceanu 2017). This can lead to disengagement within a class as students may feel that they are either ahead of the 
curve or being left behind. Both situations can lead to a lack of motivation to learn. A clear advantage of using RPGs to enhance the acquisition of a second language is that the learning is entirely in the hands of the student. Giving each student the ability to progress at their own pace increases engagement and motivation (Janebi and Haghighatpasand 2017). Furthermore, the student is free to use or ignore the learning scaffolding based on their own needs (Hanus and Fox 2015). One method employed by RPGS to enhance player motivation is the implementation of rewards systems.

In-game reward systems encourage both informal and implicit learning as the learner is able to gain both knowledge through the tasks or quests as well as tangible rewards such as achievement badges, better weapons and new avatar skins. The motivation created by wanting to gain all of the tangible achievements or the latest collectibles in order to compare them with fellow players is a driving force in the sustained gameplay of RPGs. Research by Sailer et al. (2017) also supports the theory that in-game rewards help to create positive learning experiences and add meaning to the learning tasks. These rewards form visual representations of competence and success thereby act not only as a personal motivator but also enhance competitive motivation and player autonomy.

Autonomy is present not only from the point of view of the learner but can also be witnessed in the cognitive processes behind the social collaborations and interactions (Peterson 2008; Warschauer 2005). The findings of such research into interactive CALL applications showed significant enhancements of not only the TL but also cross-cultural understanding. One of the reasons put forward for this is the anonymity created by use of player pseudonyms, meaning that it would not necessarily be easy to tell a native speaker from an L2 speaker. This aspect resonates strongly with Chinese native speakers, namely, the concept of saving face (Wen and Clement 2003). As will be observed at a later stage of this discussion, this particular premise forms one of the cornerstones of this RPG and second 
language acquisition project described later in this discussion and directly informs the avatar preference test.

\section{Research Findings: Affective Factors, Anxiety}

As is noted in the literature, certain affective filters can interrupt second language acquisition (de Haan 2005). These are the psychological factors which can inhibit language use and learning. Anxiety using the TL is a commonly reported feeling within the second language classroom, or indeed when faced with interactions with a native speaker (Ping et al. 2014; Tum 2015). Learner anxiety can exhibit itself through a reluctance to learn or progress, as well as reticence to use the language and a negative attitude within the classroom or take the form of mental blocks (Hashemi 2011). The feeling of being judged or that the level and correctness of the TL spoken is in some way an indicator of intelligence level can impact a learner's ability to learn effectively (Melchor-Couto 2017). This can display as reluctance to speak, utterances in low volume or freezing. The anxiety felt is closely related to how the language user feels they are being judged or viewed by others, native speakers or not.

Furthermore, a TL that has a significantly different culture may also exacerbate feelings of anxiety. The interactive, dynamic and fun aspects of RPGs are an intrinsic factor in reducing the anxiety a learner may feel interacting in the TL (Horowitz 2019). This resonates with Kolb's theory of experiential learning (Kolb 1981), in which the context of the situation and activity itself can lead to learning through a process of trial and reflection in order to achieve a goal. Experiential language learning may lead the user to recognise adaptability in their own TL use, feel personal growth and a greater contextual awareness of cultural differences. The findings of research by Sung et al. (2015) demonstrated that by utilising experiential gaming methods, learners displayed deep learning strategies and higher motivation levels, both of which are essential for long-term learning. In research conducted by TianJian (2010) into Chinese English language learners and anxiety, more than $50 \%$ of the 
participants stated that they experienced moderate to high anxiety when using the TL. Feelings of enhanced anxiety can also be closely associated with the concept of face.

\section{Research Findings: Avatars}

The utilization of avatars in RPGS alongside other communication media (in game text and voice chat) further enhances the immersive experience and therefore flow for the player, as the avatar can show real time physical reactions to actions. Additionally, feelings of 'telepresence' (being there) and 'co-presence' (being there with another person) are also enhanced (Peterson 2011) due to the immersive nature of games and feelings of ownership towards the avatar (McCreery et al. 2012). The avatar can be viewed as the channel for the emotional experience of play, supporting the view that an avatar can be an extension of the idealised self in the virtual world (Ratan and Sah 2015). The player/avatar relationship thus allows for higher levels of motivation.

The literature shows that avatars not only provide the opportunity for real-time interaction with native speakers but also create an arena for the development of cross-cultural competencies (Peterson 2011). Whilst the interactions in many online games may take place all across the globe, the participants all exist as avatars on the same virtual plane providing a practice arena that would otherwise be unobtainable. Thereby, they potentially enhance not only TL acquisition but also intercultural competence and contribute to the creation of a more effective platform for communication within the online environment. This further increases the sense of immersion in games and in turn cognitive flow (Nylund and Landfors 2015). As noted previously, RPG's stimulate the need of real-time avatar interaction without real-life consequences for mistakes made.

The following section examines the importance of a key element in digital gamebased language learning, namely, the importance of face. The discussion then turns to 
providing an overview of an experiment into player avatar choice preferences in a purposebuilt RPG designed specifically to facilitate language learning. In particular, how the avatar becomes the conduit for self-representation within the games space and how that is perceived by others.

\section{The Concept of Face and Relationship to Learning in Digital Games}

Face is a concept intrinsic to Chinese culture, which holds that the perception of a person and how they present themselves is potentially more important than the actual reality. The classic example of this is a Chinese student in a classroom who doesn't understand the lesson but is reluctant to ask for clarification. As this may be perceived as a clear indication of lack of knowledge which would be ascribed to a negative opinion. Whereas asking for clarification online does not elicit the same issue; in essence online, a player is faceless. This allows a degree of freedom within the interactions as there can be no perceived loss of face (Wen and Clement 2003).

Whilst the cultural concept of face may be rooted firmly in Chinese philosophy the effects are also experienced by other second language users. Goffman (1967), produced one of the first definitions of face that is applicable to all nationalities. He asserted that a person's social face is a concept derived from that person's view of their own value and role within society. If their own interpretation of their social standing is in line with how others perceive them then it can be said that that person has face. He further states that whilst a person can assume that they have a certain level of face it is actually how others view them that matters and that social value can easily be taken away should the person be found wanting or unworthy. This correlates directly to the Chinese concept of the fear of losing face. Within a language classroom how peers and native speakers view the learner has a strong influence on their anxiety, motivation and confidence to use the TL. 
When relationships are formed and maintained entirely online via the medium of avatars, the player is likely to feel less anxiety due to the 'faceless' anonymity of the interaction. Additionally, the lack of visible social prompts based on context that may otherwise cause interference, allow for a freer interaction without anxiety or inhibition (Rankin et al., 2008). This is particularly prevalent cross-linguistically between English and Mandarin. English language and social cues tend to be implicit with inference playing a large role in communication. The use of a racially neutral avatar can help to alleviate the anxiety that can be felt when communicating with a native speaker in the TL (Aymerich-Franch 2014).

The prevalence of online interactions has led to the state where social events and interactions take place solely online and with avatars acting as the conduit; being 'antisocially social' (Harbord and Dempster 2019). These proxy exchanges allow less anxiety to be experienced as the user is 'face-less' and thereby no longer constrained by the social anxiety faced when using the TL. The limited visual stimuli as well as social prompts that can be connected to interference of TL production are negated by the lack of actual face-to-face interaction. When considering communication in a second language the use of a racially neutral avatar can help to alleviate the anxiety that can be felt when communicating with a native speaker (Melchor-Couto 2017). This also helps to override the Chinese societal concept of face (Wen and Clement 2003) and the related fear of losing it.

Feeding into the negation of face is a sense of impermanence and reduction of responsibility with online relationships. For example, should a major mistake or social faux pas happen instead of trying to make amends, a new profile can be created and the cycle starts over (Nylund and Landfors 2015). This cycle can be described as 'trial and error' in which any mistakes made during the learning process can easily be amended without fear of recrimination (Hanus and Fox 2015). A contributing factor to the negation of face is the use 
of avatars. The following section outlines the pilot study on EFL learner avatar choice in an RPG noted previously.

\section{Pilot Project: Avatar Preference Test}

A pre-game test was carried out in order to tailor the RPG to the research needs and also make it more attractive to players. In order to fully assess the research hypotheses, 16 avatars were created with a variety of cultural indicators to assess the influence that avatar choice might have over the likelihood of interaction in the TL within the RPG setting. The cultural and racial indicators corresponded to the TLs as well as other races and fantasy styles. The research was directed at second language speakers and how willing or not they are to interact with avatars that represent the cultural and racial background of the TL. The choice of avatar of the TL speaker was investigated as the projection of an idealized perception. The participants were 20 Chinese students who had English as a second language and who took part in the Masters of Professional Practice Programme at Abertay University. They were given visual exposure only to a selection of avatars representing a potential TL interlocutor, with no other cues. They were then asked to express what their perception of the character was and what level of interaction they would likely take.

Second language learners reported that they experienced being more at ease when conversing with their peers within the 'safe' confines of the language classroom (Rublik 2018). However, if they are put in the position where a native speaker acts as the interlocutor, they feel anxious and unwilling to interact for fear of showing themselves up (Wen and Clement 2003). For these reasons it was expected that the choice of avatar for online interactions would follow the same pattern as a foreign language classroom.

One contributing factor is the comfort of anonymity (Roed 2010), which describes how players can interact without fear of being labelled a 'beginner' or being treated as an 
interloper in the game. For example, it was hypothesized that Caucasian Mandarin learners would be more likely to feel comfortable interacting in the TL with a more Caucasian looking avatar as opposed to an avatar that was more Asian. The reverse was also predicted for the Chinese participants. When interacting in a TL environment, players may purposely choose a more racially neutral avatar in order to disassociate themselves from the stereotype of being a second language user (Kafai et al. 2007; Lee 2014).

The aim of the research was to test three hypotheses based on avatar perception and thereby inform the larger research project into RPGs and second language acquisition. RPG maker MV (https://www.rpgmakerweb.com/products/programs/rpg-maker-mv) was used to create two identical RPGS (one in English and one in Mandarin) that could be used to test the larger hypothesis. In order to create a mutual platform with which the learners could interact online text blogs were utilised; one for English and one for Mandarin. This meant that the correspondence between the two players could be kept track of and evaluated. Additionally, it was then easier to collect data regarding the balance between English and Mandarin. For the RPG to be fully personalised to the proposed players it became necessary to design custom avatars and for that purpose player preferences were tested. For the purposes of the study, avatars with Asian and Caucasian facial features and dress were used as well as those from other cultures and fantasy. This approach formed the basis of the future planned research into RPGs and their ability to enhance the second language acquisition of both Mandarin and English. The resultant participant avatar preferences were then utilised to narrow the original 16 avatars (see Figure 1) down to 4 choices which will be provided at the beginning of the RPG. The following sections discuss the methodology and results of the avatar preference research.

Figure 1. 'Avatar choices given to the participants' 
[Insert Figure 1 here]

\section{Methodology}

The research was qualitative in nature, as the aim was to gain an awareness of the ethnographic knowledge and shared cultural preferences of each group of students. In order to achieve this goal, an online anonymous questionnaire was administered, focusing on the reasons why the participant may or may not have chosen a certain avatar to interact with. Qualitative research does leave answers open to interpretation (Allwood 2012). However, the answers given were direct enough to avoid misrepresentation.

Once the 16 avatars had been designed, an online questionnaire was created using Microsoft forms. The benefit of this format is that it was completely anonymous and thereby in line with current UK General Data Protection Regulation (GDPR) guidelines. Within the questionnaire participants were asked what their native language was, then they were asked to select one avatar for each of the three scenarios (the avatar they would choose for themselves, the avatar they would prefer to interact with and the avatar they would prefer not to interact with) and then state the reasons why they had made that choice. The participants were able to use as many or as few words as they desired to justify their responses. The questionnaire was open-ended, so they were able to take as much time as they felt necessary to complete it. The average response time was 22 minutes 15 seconds. The online questionnaire results were taken from the period from June 1, 2019 to June the 30 2019. The response was $100 \%$; a fact which may have been influenced by the researcher also being the participants' teacher.

\section{Participants}

All of the participants were given a research information sheet and consent forms and briefed about the project, and the research was carried out in line with Abertay University's code of ethics. Those who wished to take part were given access to an anonymous online 
questionnaire which presented 16 avatars and 4 corresponding questions. The participants were asked to consider the scenario where they were imagined themselves as a player in an RPG played in the TL. They were then asked to choose which avatar they would prefer to represent themselves online, which they would be more likely to interact with, and which they would not be likely to interact with.

Hypothesis 1: Participants are more likely to choose an avatar that resembles a native speaker to represent themselves.

The TL would serve as the instigator for the participant's choice. It was predicted that an avatar that corresponds racially to the TL would be chosen to represent themselves in the game. Players will select an avatar to look more racially appropriate to the TL and be more comfortable interacting with avatars that present as less racially stereotypical.

Hypothesis 2: Participants are more likely to be comfortable interacting with an avatar that is more representative of the TLs and more uncomfortable when interacting with an avatar that is more representative of their own ethnicity.

Hypothesis 3: Through the above selection of preferred avatars, participants are likely to feel less anxiety and more motivation when interacting in the TL. Additionally, the converse is also true that when faced with an avatar that the participant does not feel is attractive then they will be negatively affected in terms of motivation and willingness to communicate.

Another aspect that increases the lack of inhibition is the anonymity of gameplay. Players select an avatar which according to Peterson (2006) enhances not only the immersion and emotional investment, but also the role-play and communication between players. The use of an avatar can help negate the anxiety that a learner may feel within face-to-face conversation in the TL (Rankin et al. 2008). Peterson (2011) reported that in his research which utilised the RPG 'Allods Online' the second language learners stated that with the use 
of an avatar they were able to give themselves a new name which allowed a degree of anonymity and also a reduction in stress when speaking a second language. Which meant that they felt less fear when conversing with a native speaker.

\section{Findings}

The findings of the preference test were analysed individually by each question and linked to the corresponding hypotheses. The results did follow the original hypotheses; however, they did not match the reasons expected. Figure 2 represents the three preferred choices from the questions given in the questionnaire.

Figure 2. 'The Avatar choices in order of popularity in response to the three questions given' [Insert Figures 2a, 2b and 2c here]

\section{Q1 Q2 Q3}

Question 1 above shows the avatars that the Chinese participants were most likely to choose to represent themselves. The reasons given for these choices were: "the Chinese boy looks most like me" and "this avatar is the most neutral". The results veered slightly from hypothesis 1 in that the more culturally similar and culturally opposite avatars were chosen along with the most neutral. With a larger participant sample size a clearer picture could be garnered which would either help to prove or disprove the hypothesis.

Question 2 shows the avatars that the Chinese participants would choose to interact with in the TL. The reasons given for these selections were "She looks Scottish and I want to go to Scotland". The results from question 2 support the hypothesis that participants will be more willing to interact with an avatar that is more racially identifiable as a TL user. This is backed up by the 'thin slice' effect (Shin et al., 2019). Wherein the participant may be 
judging the veracity of the level of representation of the avatar they are interacting with based upon how well they have self-represented. Question 3 shows the avatars that the Chinese participants would not want to interact with. The reasons given for these selections were all based on aesthetics and particular cultural ideals. The results were question 3 showed the participant's motivation and willingness to interact is heavily influenced by avatar appearance.

\section{Discussion}

The following discussion will attach theoretical reasoning to the results of the findings. The results were unexpected due to the fact that online aesthetics played a much larger role in avatar selection than previously anticipated. However, this may lead back to the bias created by the selection of avatars that are more representative of the TL to interact with, with the supposition that the player may not be a native speaker.

Taylor (2002) demonstrated that for gaming, players tended to choose avatars that would either distract other players or be a close facsimile of the role that they wished to portray online. Societal norms of what is attractive can lead to the purposeful manipulation of avatars in order to present the player in a manner that elicits a far more positive response to first impressions from peers (Fong and Mar 2015; Lin and Wang 2014). Building on the premise that an avatar is a highly modified version of a person, it could then be assumed that during interactions there will be an element of dubiousness or distrust (Williams 2006). As can be seen by the results, "Identity Tourism" (Taylor 2002) appears to have taken place as the participants chose to 'try out' a new personality, appearances, races or genders.

The degree of accuracy when interpreting an avatar can be predicted by the convergence between 'cue validity' (how close to reality the avatar is to the player) and 'cue utilisation' (how the given cues are then used to create a perception of character/personality). 
The higher the convergence, then the more accurate the results. The Brunswick Lens Model of perception of personality (1956) is informed by both cue validity and utilisation. The theory states that visual clues from exposure to a stimulus (in this case an avatar) can provide a 'lens' through which non-visual conclusions can be drawn. Through this lens, predictions about the real person can be drawn from their avatar's appearance and character traits leading to thin-slice judgments that are usually accurate (Shin et al. 2019).

Additionally, when dealing with NPCs the player may also find it easier to interact with an avatar that is a closer representation of the TL as they will feel safer from judgement for mistakes made. This will allow the player a forum in which they could practice production of language in use while seeming to interact with a native speaker. In order to bypass feelings of anxiety caused by interacting with a native interlocutor (Rublik 2018; Roed 2010; Wen and Clement 2003), participants are more likely to select avatars that are less racially representative of the TL.

\section{Conclusion}

As the prior discussion on the research involving RPGS in CALL has shown, the contributory factors to learning - motivation, autonomy and real-time interaction - appear ripe for further investigation (Peterson 2008). Furthermore, research indicates the use of avatars appears to also play a significant role in learning with this type of digital game. The increasing research in the above areas may shed new light on these aspects of language learning with RPGS.

Regarding the pilot project described in this chapter, the results followed the original hypotheses. However, the justifications made for the participant choices did not match prior expectations. The Chinese participants almost all stated aesthetics to be the main influencer on their decision making. The reasoning may not be so surprising when parallels can easily be drawn between face and beauty to success and prestige. Furthermore, China has a rapidly 
growing beauty economy known as "meinü jingji" (Xu and Feiner 2007), which places greater importance on outward appearance as it strongly correlated with perceptions of character, prestige and value.

From the reasonings offered by the participants in the avatar research, the general common theme arose with regards to playing RPG's in a second language. The utilisation of avatars enhances second language acquisition by allowing for greater immersion and suspension of inhibitions. By embodying an idealised avatar, the player is free to interact with other players and NPCs without any fear of losing face. By choosing an avatar that closely represents the culture of the TL, the player is able to try out "identity tourism" (Taylor 2002). Thereby enabling them to feel less anxious when interacting with the perceived native speaker. Additionally, the anonymity afforded by the use of an avatar and screen name also appears to lower the inhibitions that might normally be faced when using the TL in front of people.

\section{Limitations}

The first limitation within the study was number of participants. Secondly, the number of avatars provided to choose from. These needed to be racially sensitive but also representative of different cultures. Ideally, the research would have the ability to allow participants to create their own avatars from a stock set of facial features and cultural identifiers. This would allow for a far more personalised response to the three questions given in the questionnaire.

One interesting factor to consider is that whilst the online questionnaire was completely anonymous, the students who took part were aware that it was for research purposes. For this reason, the researcher being Scottish could potentially have influenced the results for preferred avatars, thereby creating a confounding variable effect. 


\section{Future Directions}

The results of the avatar preference test are being used to directly inform the design and development of an RPG which aims to enhance the second language acquisition of both Mandarin and English. To that end, the game has been designed twice once in English and once in Mandarin and will have a collaborative blog between the two players of both games. The reason that an RPG was chosen refers back to the hypothesised advantages shown in table 1. Additionally, RPGs tend to have simpler game mechanics and fewer twitch reflexes, allowing the player to progress at their own pace through the game. This means they are more accessible to non-gaming players. They create immersive atmospheres where the players can lose themselves within the gameplay and not worry about the level of language produced. Moreover, they provide players with the option to read and reread their chats outside of the game allowing for consolidation and review of language used.

Although the research is on-going, it is anticipated that that the results gained from the fully completed project will demonstrate that the students who utilise the RPG to enhance their language learning will show an increase in the accuracy of understanding, production and recall of the TL. Moreover, perhaps more importantly, it is hoped that their motivation for learning and using the TL should also increase.

Figure 3. 'The preferred avatars in the context of the purpose-built RPG' [Insert Figures 3.1 and 3.2 here]

Based on the results from the avatar study, the NPCs that are encountered in the beginning of the game when the players are more nervous and less confident using the TL were replaced by the preferred choices for interaction (see Figure 3). As the player progresses through the game and the levels become harder the NPCs will be represented by the least favoured avatars. As players start to feel more confident interacting within the 
confines of the game environment it is hypothesised that they will begin to feel more comfortable with less preferred avatars.

The resultant game will help to justify the use of RPG's to enhance second language acquisition. It is hoped that it will provide enough evidence to warrant further expansion. Several areas have been identified for potential developments and expansion. One approach under consideration is the inclusion of a speech to text program for players to communicate verbally and still be able to keep a record of what was said. This would allow for a freer interaction between the players in TL conversation. Developing the game so that it can be played by two people remotely at the same time is another option, as this would enable players to combine speech or speech to text so players would be able to experience genuine real-time TL interactions. A further promising area is the incorporation of augmented reality (AR) elements to the game, which can be used to overlay TL into the real world. An investment into the narrative of the game so that it can include deeper meaning and immersive gameplay that are culturally representative of the TLs as used. Once the game is completed it will be possible to swap the languages used. One proposition is to utilize Scots Gaelic and English as a way to encourage language use between peers in a motivational and diverting way. One advantage of tailoring the game to use Scots Gaelic is the cultural preservation of a language that is not in widespread use.

Further areas for future research within this field, would allow for a greater understanding of cultural pedagogy and educational cultures. Thereby creating deeper contextual knowledge of how to approach SLA participants from different cultures. Education should not be a one size fits all approach and through an awareness of how approaches can be adapted to specific cultural variables more efficient teaching practices may be developed. 
This chapter is dedicated to Simon Harbord; scientist, inventor and best of all, Father. 1955-

2019

\section{References}

Alyaz, Y., D. Spaniel-Weise and E. Gursoy. (2017), 'A Study on Using Serious Games in Teaching German as a Foreign Language', Journal of Education and Learning, [online] 6 (3): 250-264. http://dx.doi.org/10.5539/jel.v6n3p250.

Aymerich-Franch, L., R.F. Kizilcec and J.N. Bailenson. (2014), 'The Relationship Between Virtual Self Similarity and Social Anxiety', Frontiers in Human Neuroscience, 8 (944).

Bird, S. and J. Williams. (2002). 'The Effect of Bimodal Input on Implicit and Explicit Memory: An Investigation into the Benefits of Within-Language Subtitling', Applied Psycholinguistics, 23 (4): 509-533. http://dx.doi.org/10.1017/s0142716402004022.

Blumberg, F. and E. Altschuler. (2011), 'From the Playroom to the Classroom: Children's Views of Video Game Play and Academic Learning', Child Development Perspectives, 5(2): 99-103. http://dx.doi.org/10.1111/j.17508606.2011.00163.x.

Chen, H. and T. Yang. (2013), 'The impact of adventure video games on foreign language learning and the perceptions of learners', Interactive Learning Environments, 21(2): 129-141. http://dx.doi.org/10.1080/10494820.2012.705851.

Cheng, M-T., Y-W. Lin, H-C. She and P-C. Kuo. (2017), 'Is Immersion of any Value? Whether, and to What Extent, Game Immersion Experience During Serious Gaming Affects Science Learning', British Journal of Educational Technology, 48 (2): 246-263.

Connolly, T., E. Boyle. E. MacArthur. T. Hainey. and J. Boyle. (2012), 'A systematic literature review of empirical evidence on computer games and serious games', Computers \& Education, 59 (2): 661-686. http://dx.doi.org/10.1016/j.compedu.2012.03.004.

de Haan, J. (2005), 'Learning Language Through Video Games: A Theoretical Framework, an Evaluation of Game Genres and Questions for Future Research', in S. Schaffer and M. Price (eds), Interactive Convergence: Critical Issues in Multimedia, 229-239, Oxford: Inter-disciplinary Press.

Ellis, N. (2019), 'Essentials of a Theory of Language Cognition', The Modern Language Journal, 103: 39-60. http://dx.doi.org/10.1111/modl.12532.

Felicia, P., ed. (2011), Handbook of Research on Improving Learning and Motivation Through Educational Games: Multidisciplinary Approaches, Hershey: IGI Global. 
Fong, K. and R. Mar. (2015), 'What Does My Avatar Say About Me? Inferring Personality From Avatars', Personality and Social Psychology Bulletin, 41(2): 237-249. http://dx.doi.org/10.1177/0146167214562761.

Gee, J. P. (2005), 'Learning by design: Good Video Games as Learning Machines', E-Learning and Digital Media, 2 (1): 5-16.

Goffman, E. (1967), Interaction Ritual, New York: Doubleday.

Hadfield, J. (1984), Elementary Communication Games, Hong Kong: Thomas Nelson and Sons Ltd.

Hanus, M. and J. Fox. (2015), 'Assessing the Effects of Gamification in the Classroom: A Longitudinal Study on Intrinsic Motivation, Social Comparison, Satisfaction, Effort, and Academic Performance', Computers and Education, 80: 152-161. http://dx.doi.org/10.1016/j.compedu.2014.08.019.

Harbord, C and E. Dempster. (2019), ‘Avatars: The Other Side of Proteus's Mirror', in Joint International Conference on Entertainment Computing and Serious Games, 412-416. Cham: Springer.

Hashemi, M. (2011), 'Language Stress and Anxiety Among the English Language Learners', Procedia - Social and Behavioral Sciences, 30: 1811-1816.

Horowitz, K. S. (2019), 'Video Games and English as a Second Language: The Effect of Massive Multiplayer Online Video Games on the Willingness to Communicate and Communicative Anxiety of College Students in Puerto Rico', American Journal of Play, 11 (3): 379-410.

Jabbari, N. and Z. Eslami. (2018), Second Language Learning in the Context of Massively Multiplayer Online Games: A Scoping Review', ReCALL, 31 (1): 92-113. http://dx.doi.org/10.1017/s0958344018000058.

Janebi E., M. and M. Haghighatpasand. (2017), 'Exploiting Adventure Video Games for Second Language Vocabulary Recall: A Mixed-Methods Study', Innovation in Language Learning and Teaching, 13 (1): 61-75. http://dx.doi.org/10.1080/17501229.2017.1359276

Kafai, Y., D. Fields. and M. Cook. (2007), 'Your Second Selves: Avatar Designs and Identity Play in a Teen World', in Situated Play, Los Angeles: DiGRA. http://www.digra.org/digital-library/publications/your-second-selvesresources-agency-and-constraints-in-avatar-designs-andidentity-play-in-atween-virtual-world/.

Kim, Y. (2018), 'Analysis of research trends focusing on outcomes in language learning using MMORPGs', Multimedia-Assisted Language Learning, 21 (4): 111-142.

Kolb, D. A. (1981), 'Learning styles and disciplinary differences', The Modern American College, 1: 232-255. 
Krashen, S. (1989), Language Acquisition and Language Education. Hemel Hempstead: Prentice Hall International.

Lee, J. (2014), 'Does Virtual Diversity Matter?: Effects of Avatar-Based Diversity Representation on Willingness to Express Offline Racial Identity and Avatar Customization', Computers in Human Behavior, 36: 190-197. http://dx.doi.org/10.1016/j.chb.2014.03.040.

Lee, J. and J. Pass. (2014), 'Massively Multiplayer Online Gaming and English Language Learning', in H. R. Gerber and S. Schamroth Abrams (eds), Bridging Literacies with Videogames, 91-101, Rotterdam: Sense Publishers.

Lin, H. and H. Wang. (2014), 'Avatar Creation in Virtual Worlds: Behaviors and Motivations', Computers in Human Behavior, 34: 213-218. http://dx.doi.org/10.1016/j.chb.2013.10.005

McCreery, M., K.S. Krach, P. Schrader and R. Boone. (2012), 'Defining the Virtual Self: Personality, Behavior, and the Psychology of Embodiment', Computers in Human Behavior, 28 (3): 976-983. http://dx.doi.org/10.1016/j.chb.2011.12.019

Melchor-Couto, S. (2017), 'Foreign Language Anxiety Levels in Second Life Oral Interaction', ReCALL, 29 (1): 99-119.

Nylund, A. and O. Landfors. (2015), 'Frustration and its Effect on Immersion in Games', MA diss, Umea University: Umea.

Peterson, M. (2006), 'Learner Interaction Management in an Avatar and Chat-Based Virtual World', Computer Assisted Language Learning, 19 (1): 79-103.

Peterson, M. (2008), 'Virtual Worlds in Language Education', JALT CALL Journal, 4 (3): 29-37.

Peterson, M. (2010), 'Massively Multiplayer Online Role-Playing Games as Arenas for Second Language Learning', Computer Assisted Language Learning, 23 (5): 429-439.

Peterson, M. (2011), 'Towards a Research Agenda for the Use of Three-Dimensional Virtual Worlds in Language Learning', Calico Journal, 29 (1): 67.

Peterson, M. (2012), 'Learner Interaction in a Massively Multiplayer Online Role Playing Game (MMORPG): A Sociocultural Discourse Analysis', ReCALL, 24 (3): 361-380.

Peterson, M. (2013), Computer Games and Language Learning, New York: Palgrave Macmillan.

Ping, A., D. Baranovich, M. Manueli and S. Siraj (2014), 'Promoting Self-Regulation in Vocabulary Learning among Chinese EFL Learners: A Needs Analysis', The Asia-Pacific Education Researcher, 24 (1): 137-146.

http://dx.doi.org/10.1007/s40299-013-0166-x 
Rankin, Y. A., M. McNeal, M.W. Shute and B. Gooch. (2008), 'User Centered Game Design: Evaluating Massive Multiplayer Online Role Playing Games for Second Language Acquisition', in Proceedings of the 2008 ACM SIGGRAPH Symposium on Video Games, 43-49. Los Angeles: ACM.

Ratan, R. and Y. Sah. (2015), 'Leveling Up on Stereotype Threat: The Role of Avatar Customization and Avatar Embodiment', Computers in Human Behavior, 50: 367-374. http://dx.doi.org/10.1016/j.chb.2015.04.010.

Reinhardt, J. (2019), 'Gameful Second and Foreign Language Teaching and Learning: Theory, Research, and Practice', Basingstoke, UK: PalgraveMacmillan.

Romero, M., M. Usart and M. Ott. (2015), 'Can Serious Games Contribute to Developing and Sustaining 21st Century Skills?', Games and Culture, 10 (2): 148-177.

Roed, J. (2010), 'Language Learner Behaviour in a Virtual Environment', Computer Assisted Language Learning, 16 (2-3): 155-172. http://dx.doi.org/10.1076/call.16.2.155.15880.

Rublik, N. (2018), 'Chinese Cultural Beliefs: Implications for the Chinese Learner of English', Sino-US English Teaching, 15 (4): 173-184. http://dx.doi.org/10.17265/1539-8072/2018.04.001.

Sailer, M., J. Hense, S. Mayr and H. Mandl. (2017). 'How Gamification Motivates: An Experimental Study of the Effects of Specific Game Design Elements on Psychological Need Satisfaction', Computers in Human Behavior, 69: 371380. http://dx.doi.org/10.1016/j.chb.2016.12.033.

Shin, M., S. Kim and F. Biocca. (2019),' The Uncanny Valley: No Need for any Further Judgments When an Avatar Looks Eerie', Computers in Human Behavior, 94: 100-109. http://dx.doi.org/10.1016/j.chb.2019.01.016

Sorensen, B. and B. Meyer. (2007), Serious Games in Language Learning and Teaching - A Theoretical Perspective, in DiGRA International Conference: Situated Play. Tokyo: DiGRA.

Sung, H., G. Hwang and Y. Yen. (2015), 'Development of a Contextual DecisionMaking Game for Improving Students' Learning Performance in a Health Education Course', Computers \& Education, 82: 179-190. http://dx.doi.org/10.1016/j.compedu.2014.11.012.

Suzuki, Y., T. Nakata and R. Dekeyser. (2019), 'Optimizing Second Language Practice in the Classroom: Perspectives from Cognitive Psychology', The Modern Language Journal, 103 (3): 551-561. http://dx.doi.org/10.1111/modl.12582.

Sylvén, L. K., and P. Sundqvist. (2012), 'Gaming as Extramural English L2 Learning and L2 Proficiency Among Young Learners', ReCALL, 24 (3): 302-321. 
Taylor, T. (2002), 'Living Digitally: Embodiment in Virtual Worlds', in R. Schroeder, (ed), The Social Life of Avatars: Presence and Interaction in Shared Virtual Environments, 40-62, London: Springer-Verlag.

TianJian, W. (2010), 'Speaking Anxiety: More of a Function of Personality Than Language Achievement', Chinese Journal of Applied Linguistics, 33 (5): 95109.

Topirceanu, A. (2017), 'Gamified Learning: A Role-Playing Approach to Increase Student In-Class Motivation', Procedia Computer Science, 112: 41-50. http://dx.doi.org/10.1016/j.procs.2017.08.017.

Tum, D. O. (2015), 'Foreign Language Anxiety's Forgotten Study: The Case of the Anxious Preservice Teacher', Tesol Quarterly, 49 (4): 627-658.

Warschauer, M. (2005), 'Socio-cultural perspectives on CALL', in J. Egbert and G. Petrie (eds), CALL Research Perspectives, 41-51. New Jersey: Lawrence Erlbaum.

Wen, W. and R. Clement. (2003), 'A Chinese Conceptualisation of Willingness to Communicate in ESL', Language, Culture and Curriculum, 16 (1): 18-38. http://dx.doi.org/10.1080/07908310308666654.

Williams, D. (2006), 'Virtual Cultivation: Online Worlds, Offline Perceptions', Journal of Communication, 56 (1): 69-87. http://dx.doi.org/10.1111/j.14602466.2006.00004.x.

$\mathrm{Xu}, \mathrm{G}$. and S. Feiner. (2007), 'Meinu Jingji/China's Beauty Economy: Buying Looks, Shifting Value, and Changing Place', Feminist Economics, 13 (3-4): 307-323. http://dx.doi.org/10.1080/13545700701439499.

Yang, Y. (2012), 'Building Virtual Cities, Inspiring Intelligent Citizens: Digital Games for Developing Students' Problem Solving and Learning Motivation', Computers \& Education, 59 (2): 65-377. http://dx.doi.org/10.1016/j.compedu.2012.01.012.

Zhang, Q. M. (2009), 'Affecting Factors of Native-Like Pronunciation: A Literature Review', Unpublished dissertation, Chung-Ang University, Soeul.

Zhang, Y., H. Song, X. Liu, D. Tang, Y. Chen. and X. Zhang. (2017), 'Language Learning Enhanced by Massive Multiple Online Role-Playing Games (MMORPGs) and the Underlying Behavioral and Neural Mechanisms', Frontiers in Human Neuroscience, 11. http://dx.doi.org/10.3389/fnhum.2017.00095. 